\title{
A comparative analysis of the ADAM and ADAMTS families
}

Claudia Andreini, ${ }^{1,2}$, Lucia Banci, ${ }^{1,2}$, Ivano Bertini, ${ }^{1,2, *}$, Sara Elmi, ${ }^{1}$, Antonio Rosato ${ }^{1,2}$

${ }^{1}$ Magnetic Resonance Center (CERM) - University of Florence, Via L. Sacconi 6, 50019 Sesto Fiorentino, Italy

${ }^{2}$ Department of Chemistry, University of Florence, Via della Lastruccia 3, 50019, Sesto Fiorentino, Italy

\section{SUPPORTING INFORMATION}

Table 1S: PROCHECK results (percentage of residues in the CORE, ALLOWED, GENEROUSLY ALLOWED and DISALLOWED regions of the Ramachandran plot) and PROSA Z-SCOREs for structural models and the experimental structure of ADAM17, after energy minimization in explicit solvent.

Table 2S: PROCHECK results (percentage of residues in the CORE, ALLOWED, GENEROUSLY ALLOWED and DISALLOWED regions of the Ramachandran plot) and PROSA Z-SCOREs for structural models after energy minimization in explicit solvent.

Figure 1S. Alignment of ADAMs, ADAMTS and decysin catalytic domains. Arrows show the four quite conserved cysteines. Calcium ligands and the zinc catalytic motif are highlighted in bold red and italic green, respectively (zinc ligands are box-squared).

Figure 2S. Phylogenetic tree based on the alignment of the sequences of the catalytic domains of all the protein sequences identified in the present work.

Figure 3S: Phylogenetic tree based on the alignment of the sequences of the catalytic domains of all ADAM proteins identified in the present work and decysin .

Figure 4S: Phylogenetic tree based on the alignment of the sequences of the catalytic domains of all ADAMTS proteins identified in the present work 
Table 1S: PROCHECK results (percentage of residues in the CORE, ALLOWED, GENEROUSLY ALLOWED and DISALLOWED regions of the Ramachandran plot) and PROSA Z-SCOREs for structural models and the experimental structure of ADAM17, after energy minimization in explicit solvent.

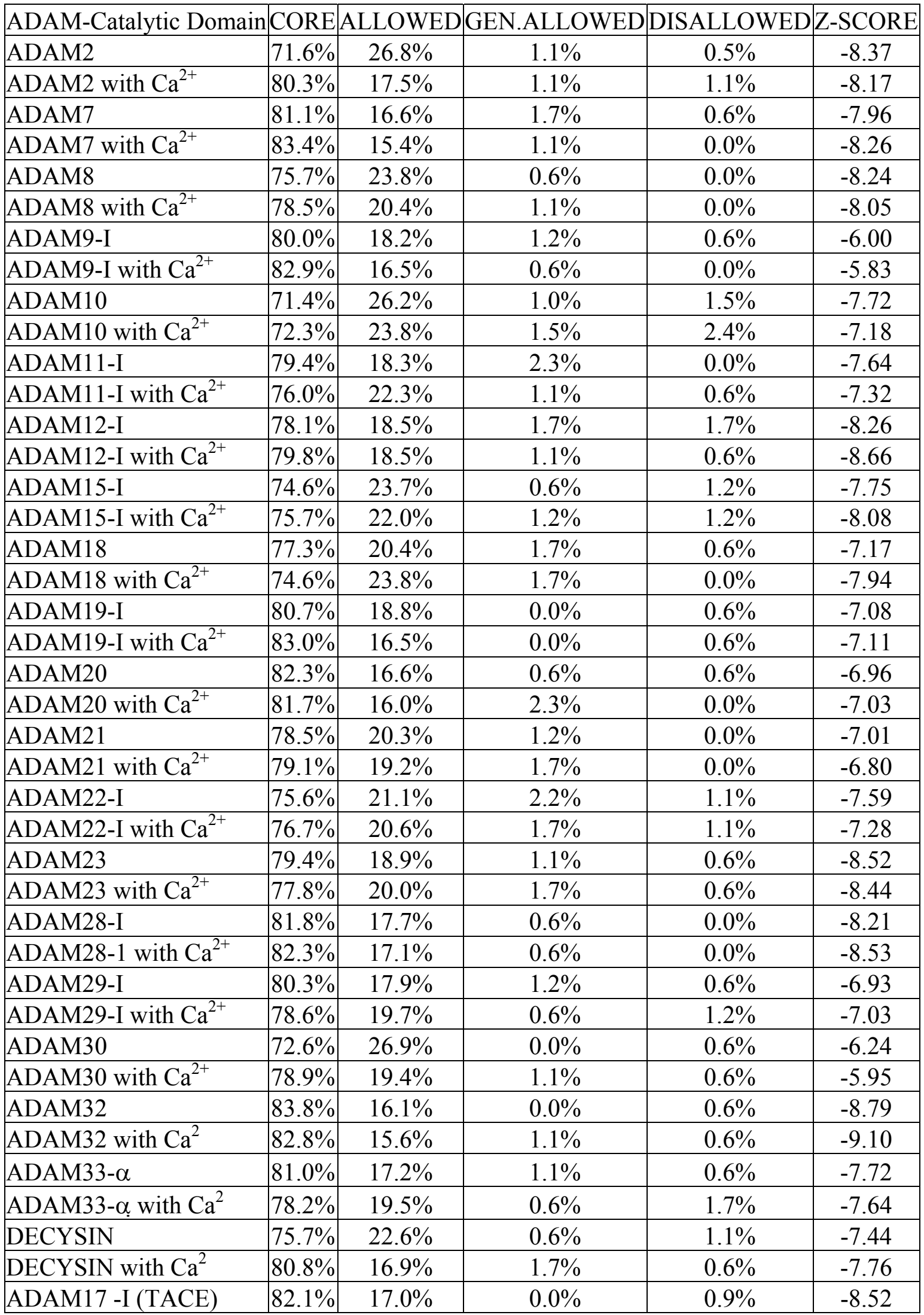


Table 2S: PROCHECK results (percentage of residues in the CORE, ALLOWED, GENEROUSLY ALLOWED and DISALLOWED regions of the Ramachandran plot) and PROSA Z-SCOREs for structural models after energy minimization in explicit solvent.

\begin{tabular}{|c|c|c|c|c|c|}
\hline ADAMTS-Catalytic Domai & CORE & ALLOWED & GEN.ALLOWED & DISALLOWED & Z-SCORE \\
\hline ADAMTS1 & $74.1 \%$ & $21.2 \%$ & $2.1 \%$ & $2.6 \%$ & -6.67 \\
\hline ADAMTS1 with $\mathrm{Ca}^{2+}$ & $75.1 \%$ & $19.6 \%$ & $4.2 \%$ & $1.1 \%$ & -5.89 \\
\hline ADAMTS2-I & $79.8 \%$ & $18.0 \%$ & $1.7 \%$ & $0.6 \%$ & -7.00 \\
\hline ADAMTS2-I with $\mathrm{Ca}^{2+}$ & $76.4 \%$ & $20.8 \%$ & $1.7 \%$ & $1.1 \%$ & -6.85 \\
\hline ADAMTS3 & $76.5 \%$ & $21.8 \%$ & $1.1 \%$ & $0.6 \%$ & -7.41 \\
\hline ADAMTS3 with $\mathrm{Ca}^{2+}$ & $74.3 \%$ & $21.2 \%$ & $2.8 \%$ & $1.7 \%$ & -7.47 \\
\hline ADAMTS4 & $77.3 \%$ & $18.8 \%$ & $1.7 \%$ & $2.2 \%$ & -6.45 \\
\hline ADAMTS4 with $\mathrm{Ca}^{2+}$ & $72.4 \%$ & $24.9 \%$ & $1.1 \%$ & $1.7 \%$ & -6.29 \\
\hline ADAMTS5 & $74.1 \%$ & $23.8 \%$ & $1.0 \%$ & $1.0 \%$ & -6.16 \\
\hline ADAMTS5 with $\mathrm{Ca}^{2+}$ & $70.5 \%$ & $26.4 \%$ & $2.6 \%$ & $0.5 \%$ & -5.46 \\
\hline ADAMTS6 & $80.6 \%$ & $18.3 \%$ & $1.1 \%$ & $0.0 \%$ & -6.08 \\
\hline ADAMTS7 & $75.4 \%$ & $23.0 \%$ & $0.5 \%$ & $1.1 \%$ & -6.81 \\
\hline ADAMTS7 with $\mathrm{Ca}^{2+}$ & $73.8 \%$ & $22.4 \%$ & $1.6 \%$ & $2.2 \%$ & -6.49 \\
\hline ADAMTS8 & $72.1 \%$ & $25.1 \%$ & $1.6 \%$ & $1.1 \%$ & -6.61 \\
\hline ADAMTS8 with $\mathrm{Ca}^{2+}$ & $67.8 \%$ & $25.7 \%$ & $3.8 \%$ & $2.7 \%$ & -7.37 \\
\hline ADAMTS9-I & $77.3 \%$ & $20.5 \%$ & $2.2 \%$ & $0.0 \%$ & -7.18 \\
\hline ADAMTS9-I with $\mathrm{Ca}^{2+}$ & $77.3 \%$ & $20.0 \%$ & $2.2 \%$ & $0.5 \%$ & -6.95 \\
\hline ADAMTS10 & $67.7 \%$ & $27.1 \%$ & $3.1 \%$ & $2.1 \%$ & -7.43 \\
\hline ADAMTS10 with $\mathrm{Ca}^{2+}$ & $70.3 \%$ & $25.5 \%$ & $2.6 \%$ & $1.6 \%$ & -7.20 \\
\hline ADAMTS12 & $76.8 \%$ & $21.1 \%$ & $1.1 \%$ & $1.1 \%$ & -7.33 \\
\hline ADAMTS12 with $\mathrm{Ca}^{2+}$ & $69.7 \%$ & $24.9 \%$ & $4.9 \%$ & $0.5 \%$ & -6.93 \\
\hline ADAMTS13-I & $76.5 \%$ & $19.4 \%$ & $2.9 \%$ & $1.2 \%$ & -7.00 \\
\hline ADAMTS13-I with $\mathrm{Ca}^{2+}$ & $75.3 \%$ & $22.4 \%$ & $2.4 \%$ & $0.0 \%$ & -7.16 \\
\hline ADAMTS13-III & $75.3 \%$ & $21.1 \%$ & $1.8 \%$ & $1.8 \%$ & -7.02 \\
\hline ADAMTS13-III with $\mathrm{Ca}^{2+}$ & $75.3 \%$ & $21.1 \%$ & $3.6 \%$ & $0.0 \%$ & -6.78 \\
\hline ADAMTS13-IV & $79.0 \%$ & $19.1 \%$ & $1.2 \%$ & $0.6 \%$ & -7.31 \\
\hline ADAMTS14-I & $79.9 \%$ & $18.4 \%$ & $0.6 \%$ & $1.1 \%$ & -6.81 \\
\hline ADAMTS14-I with $\mathrm{Ca}^{2+}$ & $71.5 \%$ & $22.3 \%$ & $3.9 \%$ & $2.2 \%$ & -7.13 \\
\hline ADAMTS15 & $77.7 \%$ & $20.7 \%$ & $0.0 \%$ & $1.6 \%$ & -6.88 \\
\hline ADAMTS15 with $\mathrm{Ca}^{2+}$ & $71.3 \%$ & $27.7 \%$ & $0.5 \%$ & $0.5 \%$ & -6.29 \\
\hline ADAMTS16 & $80.1 \%$ & $17.0 \%$ & $1.7 \%$ & $1.1 \%$ & -8.00 \\
\hline ADAMTS16 with $\mathrm{Ca}^{2+}$ & $80.7 \%$ & $15.9 \%$ & $2.8 \%$ & $0.6 \%$ & -7.67 \\
\hline ADAMTS17 & $65.8 \%$ & $30.6 \%$ & $2.6 \%$ & $1.0 \%$ & -6.32 \\
\hline ADAMTS17 with $\mathrm{Ca}^{2+}$ & $66.3 \%$ & $25.0 \%$ & $5.6 \%$ & $3.1 \%$ & -6.68 \\
\hline ADAMTS18-I & $82.8 \%$ & $17.2 \%$ & $0.6 \%$ & $0.0 \%$ & -7.58 \\
\hline ADAMTS18-I with $\mathrm{Ca}^{2+}$ & $81.0 \%$ & $17.2 \%$ & $1.1 \%$ & $0.6 \%$ & -7.35 \\
\hline ADAMTS19 & $73.1 \%$ & $24.9 \%$ & $1.5 \%$ & $0.5 \%$ & -6.99 \\
\hline ADAMTS19 with $\mathrm{Ca}^{2+}$ & $72.6 \%$ & $23.4 \%$ & $3.6 \%$ & $0.5 \%$ & -6.84 \\
\hline ADAMTS20-I & $79.4 \%$ & $18.0 \%$ & $2.1 \%$ & $0.5 \%$ & -6.30 \\
\hline ADAMTS20-I with $\mathrm{Ca}^{2+}$ & $72.5 \%$ & $24.3 \%$ & $2.1 \%$ & $1.1 \%$ & -6.43 \\
\hline
\end{tabular}


Alignment of ADAMs, ADAMTS and decysin catalytic domains. Arrows show the four quite conserved cysteines.

\section{Calcium ligands and the zinc catalytic motif are highlighted in bold red and italic green, respectively (zinc ligands are}

\section{box-squared).}

\section{ADAM2}

ADAM7

ADAM8

ADAM9-I

ADAM9-II

ADAM10

ADAM11-I

ADAM11-II

ADAM12-I

ADAM12-II

ADAM15-I

ADAM15-II

ADAM15-III

ADAM15-IV

ADAM15-V

ADAM15-VI

ADAM17-I

ADAM17-II

ADAM18

ADAM19-I

ADAM19-2

ADAM20

ADAM21

ADAM22-I

ADAM22-II

ADAM22-III

ADAM22-IV

ADAM22-V

ADAM23

ADAM28-I

ADAM28-III

ADAM29-I

ADAM29-II

ADAM29-III

ADAM 30
K Y I E M H V I V E K Q L Y N H M G S - D T T V V A Q K V F Q L I G L T N A I F V K Y V E L F I V A D D T V Y R R N - H P H N K L R N R I W G M V N F V N M I Y K $R Y V E L Y V V V D N A E F Q M L G-$ - S E A A V R H R V L E V N H V D K Y Q $R Y V E L F I V V D K E R Y D M M G R-N Q T A V R E E M I L L A N Y L D S M Y I$ $R Y V E L F I V V D K E R Y D M M G R-N Q T A V R E E M I L L A N Y L D S M Y I$

C Q L Y I Q T D H L F F K Y - - TRE A V I A Q I S S H V K A I D T I Y O T T D F $K Y V E L I V I N D H Q L F E Q M R Q-S V V L T S N F A K S V V N L A D V I Y K E--$ $K Y \vee E L I V I N D H Q L F E Q M R Q-S V V L T S N F A K S V V N L A D V I Y K E$ $K Y V E L V I V A D N R E F Q R Q G K-D L E K V K Q R L I E I A N H V D K F Y R-$ $K Y V E L V I V A D N R E F Q R Q G K-D L E K V K Q R L I E I A N H V D K F$ I $\quad$ I $K T V E L V I V A D H S E A Q K Y R--D F Q H L L N R T L E V A L L L D T F F R-$ $K T V E L V I V A D H S E A Q K Y R--D F Q H L L N R T L E V A L L L D T F F R-$ $K T V E L V I V A D H S E A Q K Y R--D F Q H L L N R T L E V A L L L D T F F R-$ $K T V E L V I V A D H S E A Q K Y R--D F Q H L L N R T L E V A L L L D T F F R-$ $K T V E L V I V A D H S E A Q K Y R--D F Q H L L N R T L E V A L L L D T F F R-$ $K T \vee E L V I V A D H S E A Q K Y R--D F Q H L L N R T L E V A L L L D T F F R$

$C K L L V V A D H R F Y R Y M G R G E E S T T T N Y L I E L I D R V D E I F R N$

$C K L L V V A D H R F Y R Y M G R G E E S T T T N Y L I E L I D R V D D I N R N-$ Q Y L E I Y I I V E K A L Y D Y M G S - E M M A V T Q K I V Q V I G L V N T M F T $K Y V E L Y L V A D Y L E F Q K N R R-D Q D A T K H K L I E I A N Y V D K F Y R-$ $K Y V E L Y L V A D Y L E F Q K N R R-D Q D A T K H K L I E I A N Y V D K F Y R-$ R F V E L V V V V D I R Y L F S Q S - N A T T V Q H E V F N V V N I D S F Y H W F L E L V V V V N H D F F I Y S Q S - N I S K V Q E D V F L V V N I V D S M Y Q $K Y I E L M I V N D H L M F K K H R L-S V V H T N T Y A K S V V N M A D L I Y K D$ $K Y I E L M I V N D H L M F K K H R L-S V V H T N T Y A K S V V N M A D L I Y K D$ $K Y I E L M I V N D H L M F K K H R L-S V V H T N T Y A K S V V N M A D L I Y K D$ $K Y I E L M I V N D H L M F K K H R L-S V V H T N T Y A K S V V N M A D L I Y K D$ $K Y I E L M I V N D H L M F K K H R L-S V V H T N T Y A K S V V N M A D L I Y K D$ $K Y L E L M I V N D H K T Y K K H R S-S H A H T N N F A K S V V N L V D S I C K E$ $K Y$ I E Y Y L V L D N G E F K R Y N E - N Q D E I R K R V F E MA N Y V N M L Y K $K Y$ I E Y Y L V L D N E F K R Y N E - N Q D E I R K R V F E M A N Y V N M L Y K $R$ I V E I V V V I D N Y L Y I R Y E R - N D S K L L E D L Y V I V N I V D S I L D $R$ I V E I V V V I D N Y L Y I R Y E R - N D S K L L E D L Y V I V N I V D S I L D $R$ I V E I V V V I D N Y L Y I R Y E R - N D S K L L E D L Y V I V N I V D S I L D K Y L E L I L L F D Q S R Y R F V N N - N L S Q V I H D A I L L T G I M D T Y F Q -
S F N I T I I L S S L E L W I $T L N$ I $H V T$ T $V$ G I E I W T $K L N F R V V L V G L E I W N$ $M L N I R$ I $V L V G L E I \quad W T$ $M L N$ I $R$ I $V$ L $V$ V G L E I W T G I R N I S F M V K R I R I $Q L N T R I V L V A M E T W A$ $Q L N T R I V L V A M E T W A$ $P L N I R$ I $V L V G \vee E V W N$ $P L N$ I R I V L V G V E V N - $P L N \vee R \vee A L V G L E A W T$ $P L N \vee R \vee A L V G L E A W T$ $P L N \vee R \vee A L V G L E A W T$ $P L N \vee R \vee A L V G L E A W T$ $P L N \vee R \vee A L V G L E A W T$ $P L N \vee R \vee A L V G L E A W T$ $T S W D N A G F \quad K G Y G \quad Q \quad Q \quad$ I $T S W D N A G K G Y G I Q I$ Q F K L T V I L S L E L W S $S L N$ I R I A L V G L E V W T $S L N$ I $R$ I A L V G L E V W T

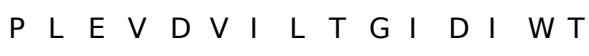
$Q L G T$ I I I L I G I E I W N $Q L K T R I V L V A M E T W A$ $Q L K T R I V L V A M E T W A$ $Q L K T R I V L V A M E T W A$ $Q L K T R I V L V A M E T W A$ $Q L K T R I V L V A M E T W A$ Q L N T R V V L V A V E T W T $K L N T H \vee A L V G M E I \quad W T$ $K L N T H \vee A L V G M E I W T$ $V$ I GV KV L L F G L E I W T $V$ I G V K V L L F G L E I W T $V$ I $G \vee K \vee L L F G L E I N T$ $D V R M R I H L K A L E V W T$ 
L Y L E M H IVVDKTLD- Y WGS - D S M IVTNKVIEIVGLANSMFT- - - QF KVTIVLSSLELWS K Y L E L Y I VADHTLFLTRHR - NLNHTKQRLLEVANYVDQLLR- - - TLDIQVALTGLEVWT KY $Y$ L L Y I VADHTLFLTRHR - NLNHTKQRLLEVANYVDQLLR- - - TLDIQVALTGLEVWT $K Y I D L Y L V L D N A F Y K N Y N E-N L T L I R S F V F D V M N L L N V I Y N-\cdots-$ I I DVQVALVGMEIWS R Y V E T MLVADQSMAEFH- - - GSGLKHYLLTLFSVAARLYKHPSIRNSVSLVVVKILVIH - I I V L L GVDDSVVQFHG- - KEHVQKYLLTLMNIVNEIYHDESLGAHINVVLVRIILLS - I I V L L GVDDSVVQFHG- - KEHVQKYLLTLMNIVNEIYHDESLGAHINVVLVRIILLS - - I EVLLGVDDSVVRFHG- - KEHVQNYLLTLMNIVNEIYHDESLGVHINVVLVRMIMLG R F V E T L V VADDKMAAFH- - - GAGLKRYLLTVMAAAAKAFKHPSIRNPVSLVVTRLVILG $R Q V E L L L V A D A S M A R L Y-\ldots$ - G R G Q $R Y L L T L A S I A N R L Y S H A S I E N H I R L A V V K V V V L G$ $R F V E T L V V A D K M M V G Y H G-\ldots R$ - R I E H Y I L S V M N I VAKLYRDS SLGNVVNIIVARLIVLT $K W V E T L V V A D A K M V E Y H G-\cdots P P Q E S Y V L T I M N M V A G L F H D P S I G N P I H I T I V R L V L L E$ RFVETLLVADASMAAFY- - - GADLQNHILTLMSVAARIYKHPSI KNSI NLMVVKVLIVE $R F V E V L V V A D N R M V S Y H \cdots-$ - G E N L Q H Y I L TLMSIVASIVK D P S I GNLINIVIVNLIVIH

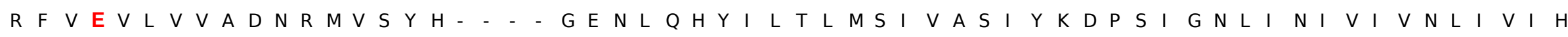

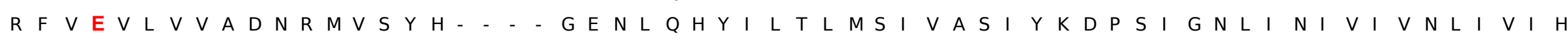
$R Y V E T L V V A D K M M V A Y H G-\cdots R R D V E Q Y V L A I M N I V A K L F Q D S S L G S T V N I L V T R L I L L T$ $R W V E T L V V A D T K M I E Y H G-\cdots S E N V E S Y I L T I M N M V T G L F H N P S I G N A I H I V V V R L I L L E$ L H L E L L VAVGPDVFQAH- - - Q E D TERYVLTNLNIGAELLRDPSLGAQFRVHLVKMVILT L H L E L L V A V G P D V Q A - - - Q E D TERYVLTNLNIGAELLRDPSLGAQFRVHLVKMVILT L H L ELLVAVGPDVFQAH- - - Q E D TERYVLTNLNIGAELLRDPSLGAQFRVHLVKMVILT L H L ELLVAVGPDVFQAH- - - Q E D TER Y V L T NLN I GAELLRDPSLGAQFRVHLVKMVILT - I E V LLVVDDSVVRFHG- - KEHVQNYVLTLMNIVDEIYHDESLGVHINIALVRLIMVG - - I EVLLVVDDSVVRFHG- - K E HVQNYVLTLMNIVDEIYHDESLGVHINIALVRLIMVG R Y VETLVVADESMVKFH- - - GADLEHYLLTLLATAARLYRHPSILNPIVIVVVKVLLLR - - VETLVVVDKKMMQNHG- - HEN I T T Y VLTILNMVSALFKDGTIGGNI N I A IVGLILLE H T VETLVVADADMVQYHG- - A EAAQRFILTVMNMVYNMFQHQSLGIKINIQVTKLVLLR - - VETLVVADKKMVEKHG- - K KNVTTYILTVMNMVSGLFKDGTIGSDINVVVVSLILLE - - VETLVVADKKMVEKHG- - K GNVTTYILTVMKVS-GLFKDGTIGSDIVNVVVVSLILLE - - I E T VVVADPAMVSYHG- - A D A A R R I L T I L N MVFNLFQHKSLGVQVNLRVIKLILLH $R Y I E I M V T A D A K V V S A H-\cdots G S N L Q N Y I L T L M S I V A T I Y K D P S I G N L I H I V V V K L V M I H$ R Y I E I MVTADAKVVSAH- - G S N L Q N Y I L TLMSIVATI Y K D P S I GNLI HIVVVKLVMI H 
$D E N K$ I A T T G -

E A N E L L H T F L R W K T S Y L V L R

N I $E$ T T L L R F S F W Q E K I L K T $R$ $S Q D R F H V P$

$N G N L I N$ I $V G$

$N G N L I N$ I $V$ G $N$ T T A D E K D P

$D G D K I Q V Q D$

$D G D K I \quad Q \vee Q D$

$D M D K C S \vee S Q$

$D M D K C S \vee S Q$

$Q R D L V E I S P$

Q R D L V I S P

$Q R D L V E I S P$

Q R D L V I S P

Q R D L V I S P

$Q R D L V E I S P$

E $P$ I L L S P Q $N E N$ I S T S G

$H$ G N M E V S E

$H$ G N M C E V S E

A S N P L P T S G

Q G N V F P M T -

$T$ D N K A I S E

$T D N K F A I S E$

$T D N K F A I S E$

$T D N K F A I S E$

$T$ D N F A I S E

E K D Q I $\quad D \quad I \quad T \quad T$

$D \quad K \quad D \quad K \quad I \quad K \quad I \quad T \quad P$

$D K D K I \quad K \quad I \quad T \quad P$

$N$ K N L I V V D -

$N K N L I V V D-$

$N$ K N L I $\vee \vee D$

D F N K I R V G Y P

$D E N K I S T V G$.

E R D R S R T Q

E R D R S R V T Q

$D G D K I K V \vee P$
D P S V T L E N L L T W Q A R Q R T R R

$G A G D V L G N F \vee Q W R$ K $F$ L I T R

$G A G D V L G N F \vee Q W R E K F L I T R$

$P F R F P N$ I G V E K F L E L N

$D L L E T L A R L M V Y R R E G L P$ -

D P F T S L HE F L D W K M K L L P R

$D P F T S L H E F L D W R K M K L L P R$

N P A V T L E N F H WR R H L L P R

N P A V T L E N F L H WR R A H L L P R

N P A V T L E N F L H WR R A H L L P R

N P A V T L E F L H WR R A H L $L R$

N P A V T L E N F H WR R H L L P R

N P A V T L E N L H WR R A H L P R D A D D I L Q R F L A W K R D Y L I L R $N$ P Y S T L W S F L S WR R K - L L A Q N P Y S T L W S F S WR R K - L L A Q $D L D N V L E D F S I W K N Y N L N N R$ $S$ I $E$ Q V L N D F S Q W K Q I S L S - Q N P L I T L R E F M K Y R R D F I K $N P L I T L R E F M K Y R R \quad D F I K$. $N P L I T L R E F M K Y R R D F I K-$ $N$ P L I T L R E F M K Y R R D F I K $N$ P L I T L R E F M K Y R R D F I K $N P \vee Q M L H E F S K Y R Q R-I K$ $N$ A S F T L E N S K WR G S V L $S R$ $N$ A S F T L E N F S KWR G S V L S R $D \vee R K S V H L Y C K W K S E N I T P R$. $D V R K S V H L Y C K W K S E N I T P R$. $D V R K S V H L Y C K W K S E N I T P R$ E L A E V L G R F V I Y K K S V L N A R E A D E L L Q K F L E W K Q S Y L N L R $D A N A T L W A F L Q W R R G-L W A Q$. D A N A T L W F L Q WR G - L W A S A S T T F D F L R W H S N L G - K
$P$ H D K D F D $\mathrm{H}$ L $\mathrm{H}$ D $\mathrm{R} R \mathrm{H} \mathrm{D}$ $\mathrm{R} R \mathrm{H}$ Q N H D D Y - E P S D E P S N K S H D K S H D $L P H D$ $L P H D$ $L P H D$ $L P H D$ $L P H D$ $L P H D$ $F S F D I A E E$ F S F I A E E - $\mathrm{PHD}$. K Y H D K Y H D L Q H D L Q H D E K S D E K S D E K S D E K S D E K S D Q H A D $\mathrm{K} R \mathrm{H}$ $\mathrm{K} \mathrm{R} \mathrm{H}$ $M Q H D$ $M Q H D$ $M Q H D$ L S S D - $P H D$ R P H R P H K I H D 
ADAMTS1 ADAMTS2-I ADAMTS2-II ADAMTS3 ADAMTS4 ADAMTS5 ADAMTS6 ADAMTS7 ADAMTS8 ADAMTS9-I ADAMTS9-II ADAMTS9-III ADAMTS10 ADAMTS12 ADAMTS13-I ADAMTS13-II ADAMTS13-III ADAMTS13-IV ADAMTS14-I ADAMTS14-II ADAMTS15 ADAMTS16 ADAMTS17 ADAMTS18-I ADAMTS18-II ADAMTS19 ADAMTS20-I ADAMTS20-II
D E Q K G P E V T -

S N A A L T L R N F C N W Q K Q H N P P S -

D R D A H - -

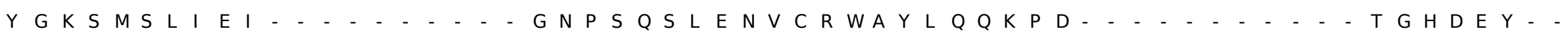

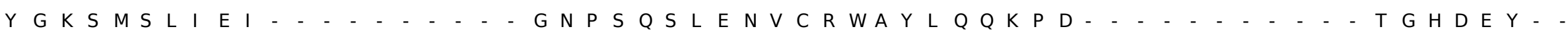

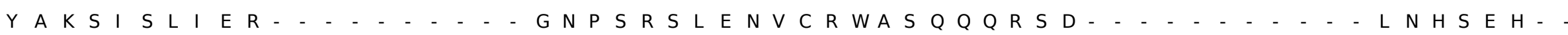

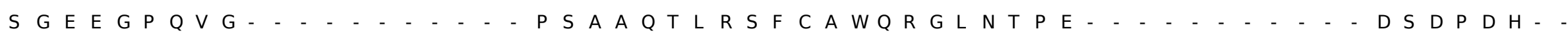

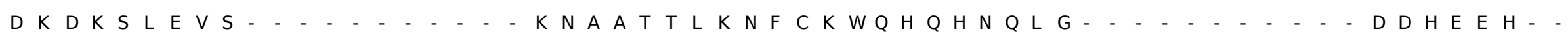

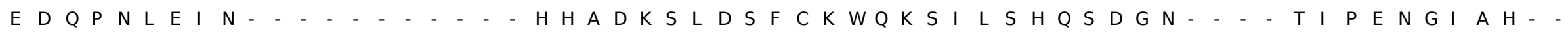

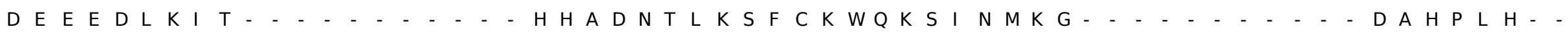

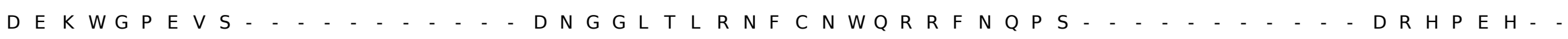

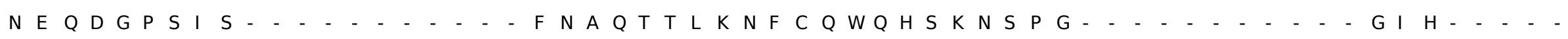

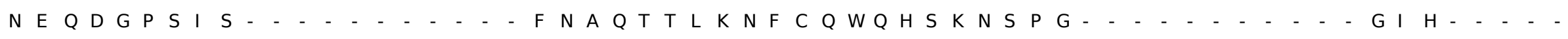

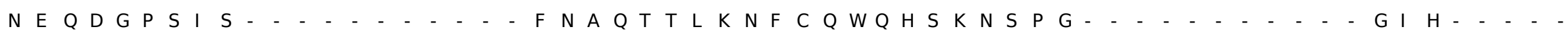

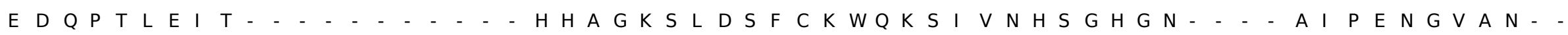

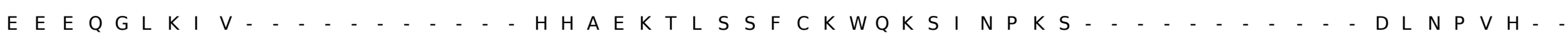

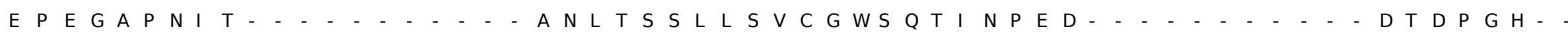

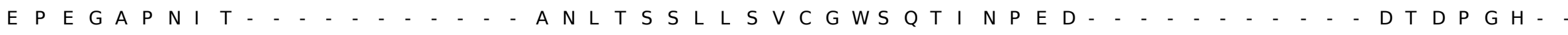

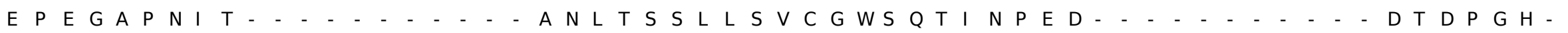

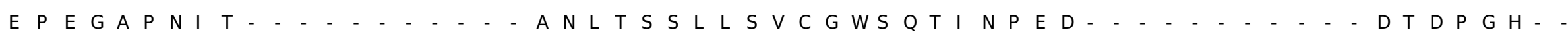

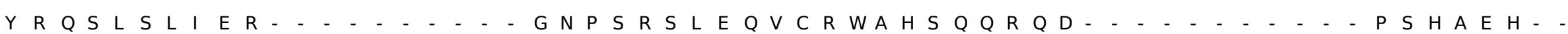

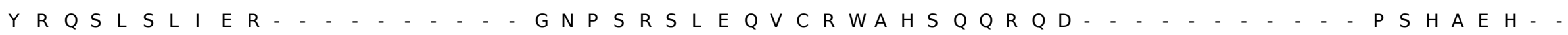

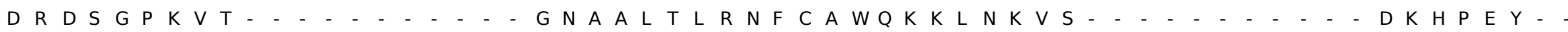

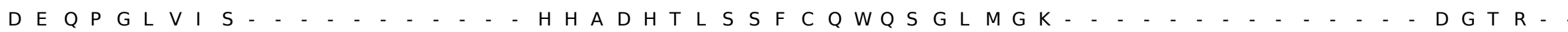

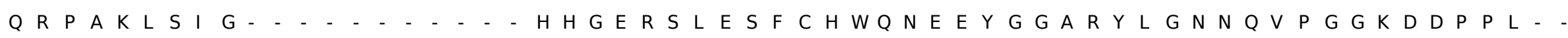

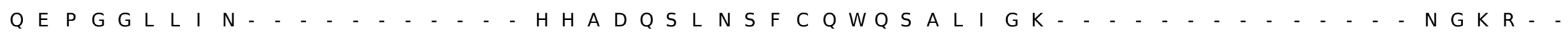

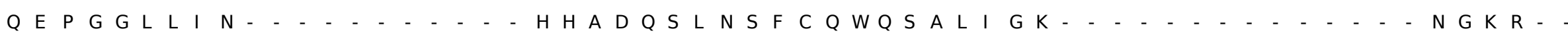

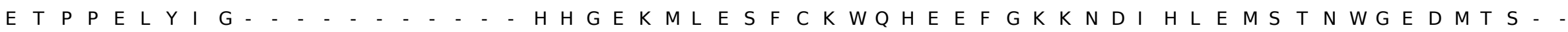

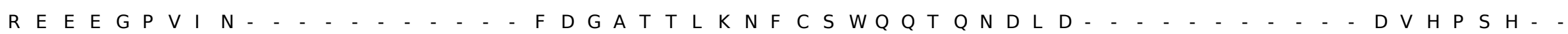

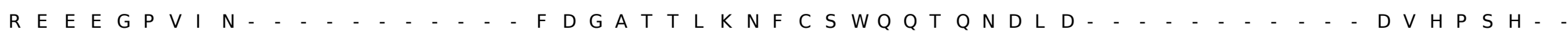


L L S G K W L Y S $S A Q L V L K K G F G$ $S A Q L V L K K G F G G$ -

C L A Y V F T D R D F D G A THLFSGRTF QS A THLFSGRTF QS N A Q L VS GVYF Q G N A Q L VS GVYF Q G S A Q L V T G T S F G $S A Q L V T G T S F S G$. $S A Q L V T G T S F S G$ $S A Q L V T G T S F S G$ $S A Q L V T G T S F S G$ $S A Q L V T G T S F S G$ A S KVCLAHLFTYQDF D M . A S KVCLAHLFTYQDFDM I A Y L L V Y K H P K NAQL I T GMSFHG NA Q L I T GMSFHG VAHLF I K D T Q G M A A H M I KNSLIS $A \vee H L F S G S Q F E S$ $A \vee H L F S G S Q F E S$ A $V H L F S G S Q F E S$ $A \vee H L F S G S Q F E S$ $A \vee H L F S G S Q F E S$ A V H L I S R V T H Y I A Q L I T A TELA G I A Q L I T A T E A G $T S H L F T$ T L G L R G $T S H L F T T L G L R G$ T S H L F T T L G L R G W A H L Y L Q R K Y N I A Y L I Y M D Y P R $S A Q L L T G R A F Q G$ $S A Q L L T G R A F Q G$ $H A Q L L S G I S F N ~ N$
$H \vee Q G I S Y P G$

$T T \vee G F A R \vee S$

TA $T$ A A $V$ V

$T A G M A F \vee G$

$V L G L A W V G A P S G S S-G G I C E K S K L Y S D G K K K S-L N$

$T S S$ G A A $Y V G$ -

$T$ T I G M A P I M -

$T T$ I G M P I $M$

$P T \vee G M A I Q N$

$P T \vee G M A I Q N$

$P T V G M A I Q N$

$P \quad T \vee G M A I Q N$

$P T \vee G M A I Q N$

$P T \vee G M A I Q N$

$G T L G L A Y V G S P R A N S H G G \vee C P K A Y Y S P V G K K N I Y L N$ $G T L G L A Y V G S P R A N S H G G V C P K A Y Y S P V G K K N I C L N$ - $Y V G A T F P G$ $T T I G L A P L M$ -

$T T I G L A P L M-$

$K L G \vee A Y V K$

I L G L A Y A

$S R S G A A Y I G$

$S R S G A A Y I G$

$S R S G A A Y I G$

$S R S G A A Y I G$

$S R S G A A Y I G$

$K R S S L S Y F G$

$T T V G L A F M$

$T T V G L A F M S$

$\begin{array}{llllllll}L & S & G & I & G & A & F & R \\ L & S & G & I & G & A & F & R\end{array}$

$L S G I G A F R$

D A L A W F G

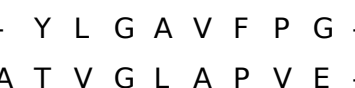

A T V G A P V E

$R R V L A A S N$

\begin{tabular}{|c|c|c|c|c|c|c|c|c|c|c|c|c|c|c|c|}
\hline & V & $C N$ & $N \mathrm{~K}$ & $\mathrm{~s}$ & & & & D & & $\mathrm{G}$ & & & & & \\
\hline$A$ & M & C S & $S \mathrm{~V}$ & $Y$ & $\mathrm{Q}$ & - & - & $\mathrm{S}$ & G & $\mathrm{G}$ & - & - & - & - & \\
\hline & $\mathrm{M}$ & $\mathrm{CS}$ & $S \mathrm{~V}$ & $Y$ & $Q$ & - & - & $\mathrm{S}$ & $\mathrm{G}$ & G & - & - & - & - & \\
\hline & & $C \mathrm{C}$ & $\mathrm{QN}$ & $P$ & $\mathrm{~F}$ & - & - & $\mathrm{N}$ & $\mathrm{T}$ & G & - & - & _. & - & \\
\hline & & $C R$ & $\mathrm{R} P$ & $P$ & I & - & - & $D$ & C & $\mathrm{G}$ & - & 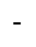 & 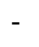 & - & \\
\hline 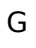 & 1 & $\mathrm{CS}$ & $S \mathrm{~L}$ & L & $\mathrm{K}$ & 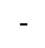 & - & $\mathrm{G}$ & G & G & - & - & 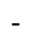 & - & \\
\hline$G$ & & C S & $S \mathrm{~L}$ & L & $\mathrm{K}$ & - & - & G & G & $\mathrm{G}$ & - & - & - & - & \\
\hline $\mathrm{G}$ & I & $\mathrm{CS}$ & $S \mathrm{~L}$ & L & $\mathrm{K}$ & - & - & $\mathrm{G}$ & G & $\mathrm{G}$ & - & - & - & - & \\
\hline G & & C S & $S \mathrm{~L}$ & $L$ & $\mathrm{~K}$ & - & - & G & G & G & - & - & - & - & \\
\hline $\mathrm{G}$ & 1 & C S & $S \mathrm{~L}$ & L & $\mathrm{K}$ & - & - & $\mathrm{G}$ & G & $\mathrm{G}$ & - & - & - & - & \\
\hline G & V & $\mathrm{CS}$ & $S R$ & $\mathrm{~T}$ & $\mathrm{R}$ & - & - & G & $\mathrm{V}$ & $\mathrm{G}$ & - & - & - & - & \\
\hline 1 & M & $\mathrm{CS}$ & $S P$ & $Y$ & - & - & - & $\mathrm{S}$ & $\mathrm{V}$ & G & - & - & - & - & \\
\hline T & M & $\mathrm{CS}$ & $S P$ & $Y$ & - & - & - & $\mathrm{S}$ & V & G & - & - & - & - & \\
\hline G & M & C T & $\begin{array}{lll}T & P\end{array}$ & $\mathrm{H}$ & $\mathrm{R}$ & - & - & $\mathrm{S}$ & C & $A$ & - & . & - & - & \\
\hline G & M & C T & $\begin{array}{ll}T & P\end{array}$ & $\mathrm{H}$ & $\mathrm{R}$ & - & - & $\mathrm{S}$ & C & $A$ & - & - & - & - & \\
\hline G & M & C T & $\begin{array}{cc}T & P\end{array}$ & $\mathrm{H}$ & $\mathrm{R}$ & - & - & $\mathrm{S}$ & C & A & - & - & - & - & \\
\hline K & V & C S & $S \mathrm{~L}$ & E & $Y$ & $A$ & $\mathrm{G}$ & $\mathrm{S}$ & $\mathrm{V}$ & $\mathrm{S}$ & - & 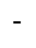 & - & - & \\
\hline $\mathrm{T}$ & M & C I & $\mathrm{I} \quad \mathrm{T}$ & $\mathrm{R}$ & $\mathrm{Y}$ & - & - & $\mathrm{S}$ & $A$ & $\mathrm{G}$ & - & - & - & - & \\
\hline G & M & $C \mathrm{R}$ & $\mathrm{R} A$ & $E$ & $\mathrm{~S}$ & - & - & $\mathrm{S}$ & $\mathrm{G}$ & & - & - & - & - & \\
\hline G & M & $C R$ & $\mathrm{R} A$ & $E$ & $\mathrm{~S}$ & - & - & $\mathrm{S}$ & $\mathrm{G}$ & $\mathrm{G}$ & 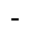 & - & - & - & \\
\hline$S$ & L & C S & $S P$ & $S$ & - & - & & $\mathrm{S}$ & & & & & & - & \\
\hline
\end{tabular}

G I C S L S H - G G G

$S M C T A D Q$ - S G

$S M C T A D Q-$ - S G G .

$S$ I $S P D F$ - S G G .

SI C S P D F - $S G G$

$S$ I $S P D F-$ - S G G .

$S$ I C $S D F$ - S G G -

$S$ I C S DF - S G G .

Page 5 


\section{ADAMTS1} ADAMTS2-I ADAMTS2-II ADAMTS3 ADAMTS4 ADAMTS5 ADAMTS6 ADAMTS7 ADAMTS8 ADAMTS9-I ADAMTS9-II ADAMTS9-III ADAMTS10 ADAMTS12 ADAMTS13-I ADAMTS13-II ADAMTS13-III ADAMTS13-IV ADAMTS14-I ADAMTS14-II ADAMTS15 ADAMTS16 ADAMTS17 ADAMTS18-I ADAMTS18-II ADAMTS19 ADAMTS20-I ADAMTS20-II
Y D T A I L F T R Q D L C G - S Q T - C D - T L G M A V G -

H D H A I F L T R Q D G - P S G - - - MQGYAPVT- - - - - -

- - HDHAIFLTRQDFG-PSG- - - MQGYAPVT-

H D H A I F L T R Q D F G P A G - - - MQ G Y A P V T -

F D T A I L T R Q D L C - VST-CD - TLGMADVG

$Y D A A I L F T R E D L C G-H H S-C D-T L G M A D V G$

$H D T A I L L T R K D L C A A M N R P C E-T L G L S H V A-\ldots$

$Y D T A I L L T R Q N F C G Q E G L-C D-T L G \vee A D I G-$

$H D T A V L L T R Q D I C R A H D K-C D-T L G L A E L G$

$H D T A V L L T R Q D I C R A H D K-C D-T L G L A E L G-$

$H D T A V L I T R Y D I C I Y K N K P C G-T L G L A P V G-$

$H D V A V L L T R K D I C A G F N R P C E-T L G L S H L S-$

$A D L V L Y I T R F D L E L P D G N-R Q-V R G \vee T Q L G$

$A D L V L Y I T R F D L E L P D G N-R Q-V R G \vee T Q L G-$

$A D L V L Y I T R F D L E L P D G N-R Q-V R G \vee T Q L G$

$A D L V L Y I T R F D L E L P D G N-R Q-V R G V T Q L G-$

H D H V V F L T R D F G P S - - - M Q G Y A P T .

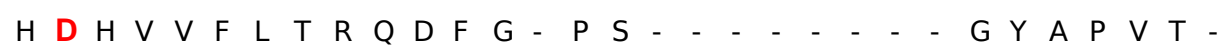

WD T A I L F T R D L C - A T T - C D - T L G M D V -

$H$ D H A I L T G L I C S W K N E P C D - T L G F A P I S -

V D A A V F T R T D C V H K D P C D - TVG I A Y L G -

H D H I L L T G F I C S WKNEP C D - T L G F A P I S -

$H D H A I L L T G F I C S W K E P C D-T L G F A P I S-$

V D A A I L I TR K D F C V H K D E P C D - T V G I A Y L S -

$H D T A V L I T R Y D I C S S K E K-C N-M L G L S Y L G-$

$H D T A V L I T R Y D I C S S K E K-C N-M L G L S Y L G-$
G M C H P R - S C T -

$-\quad-$

G MCHPVR - S C T - - - - -

$G M C H P \vee R--S C T$.

$T V C D P A R--S C A$.

$G M C E P E R--S C S-\cdots$

GMCQPHR - - SCS - - - -

T I CDPNK- - S C S - - - -

T I CDPYR - S C S - - - -

T I CDPYR - S C S - - - - -

$T$ I CDPYR - S C S - - - - GMCERER - - SCS - - - - $G M C Q P H R--S C N-\cdots$ GACSPTW- $\mathrm{S} C \mathrm{C}$ - - - - GACSPTW- $\mathrm{S} C \mathrm{C}$ - - - - -

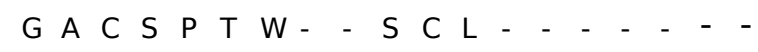
G A C S P T W - SCL - - - - $G M C H P L R$ - SCA - - - GMCHPLR - S SA - - - - $T M C D P K R$ - $S C S-S_{-}-{ }_{-}$ GMCSKYR - $S C T-\mathrm{C}_{-}-\mathrm{C}^{-}-$ GVCSAKR - K K V - - - - GMCSKYR - - SCT - - - - GMCSKYR - $S C T-T_{-}-{ }_{-}-$

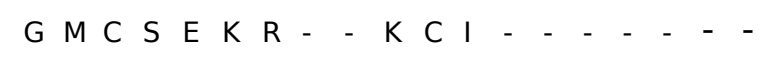
T I C D P Q - S C F - - - T I C D P Q - S C F - - - - 


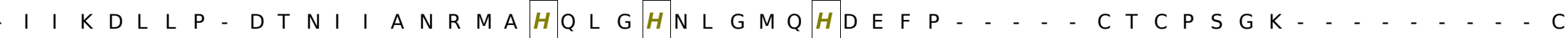
- - - - V V Q D - S K N P V G V A T MAHEMGHNLGMDHDEN- - V Q GCRCQERFEA- - - G R C - - - I N V F G - Q I TVETFASIVAHELGHNLGMNHDDGRD. I N V F G - Q I TVETFASIVAHELGHNLGMNHDDGRD.

$T G I I T \vee Q N Y G S H \vee P P K \vee S H I T F A H E \vee G H N F G S P H D S G$

C S C G A K S -

C S C G A K S -

TECTPGESKNLGQKENGNY $V N E Y G-\ldots N M G A M A V T L A Q T L G Q N L G M M W N K H R S S A G D C K C P D I W L \cdots \cdots$

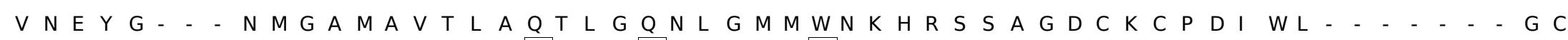
I V M D H - S D N P L A A V T L A HELGHNFGMNHDTL- - D R GCSCQMAVEK- - - GGC IVMDH-SDNPLGAAVTLAHELGHNFGMNHDTL- - D R GCSCQMAVEK- - - G G C $\checkmark N M D H-S T S I L G \vee A S S I A H E L G H S L G L D H D L P-\cdots$ - G S C P C P GPAPA- - - K K T C

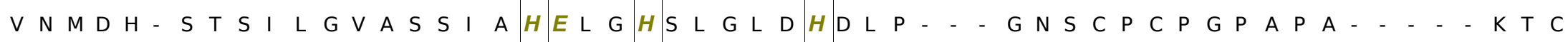

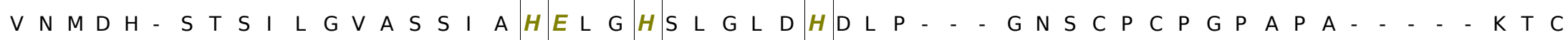
VNMDH-STSILGVASSIAHELGHSLGLDHDLP- - G N S C P C P G P A P A - - - K T C VNMDH-STSILGVASSIA HELGHSLGLDHDLP- - G N S C P C P GPAPA- - - K K T VNMDH-STSILGVASSIAHELGHSLGLDHDLP- - G N S C P C P G P A P A - - - K T C S GLTSTKNYGKTILTKEADLVTTHELGHNFGAEHDPDG- L A E C A P NEDQG- - - - G K Y S GLTSTKNYGKTILTKEADLVTTHELGHNFGAEHDPDG- L A E C A P N E D Q G - - - - GKY I A MYPDA I GLEGFSVI I A Q L L GLNVGLTYDDITQ - - - C C F C R A T - . - . - C C $\vee N M D H-S E N A I G \vee A A T M A H E M G H N F G M T H D S A-\cdots D-C C S A S A A D-\cdots$ $\checkmark N M D H-S E N A I G \vee A A T M A H E M G H N F G M T H D S A-\ldots D-C C S A S A A D-\cdots$

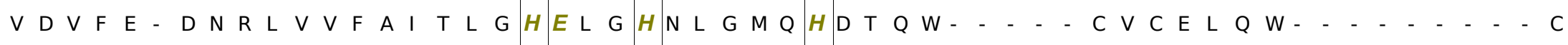

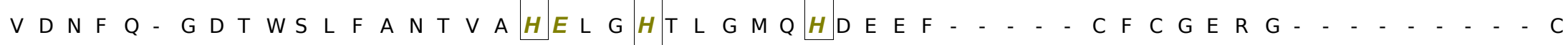

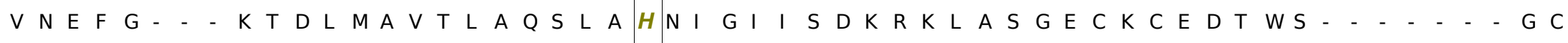

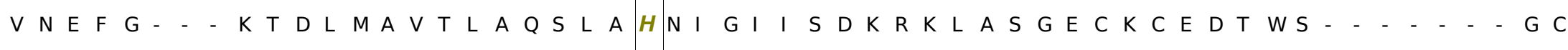
VNEFG- - KT DLMAVTLAQSLAHNIGIISDKRKLASGECKCEDTWS- - - - - G C

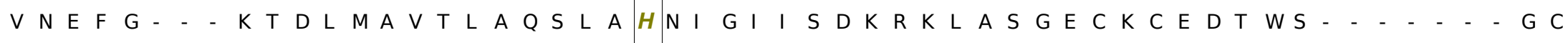
VNEFG- - K K D L MAVTLAQSLAHNIGIISDKRKLASGECKCEDTWS- - - - - G C VNEYG- - L P MAVAQVLSQSLAQNLGI QWEPS - SRKPKCDCTESWG- - - - - G C $\vee V Q D H-S D N L L R \vee A G T M A H E M G H N F G M F H D D Y-\ldots$ - - C K C P S T - - - - - - C $\vee \vee Q D H-S D N L L R \vee A G T M A H E M G H N F G M F H D D Y-\cdots S-C K C P S T I-\cdots$

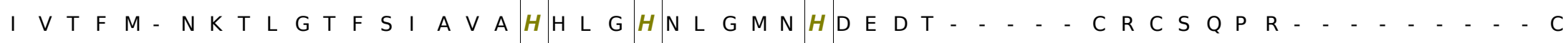

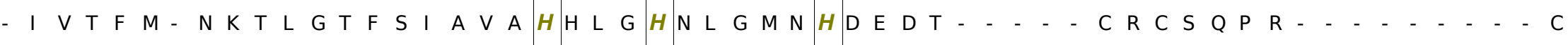
I V T F M - NKTLGTFSI A V A H H L GNLGMNHDEDT- - - C R C S Q P R - . - . - C TLLDT- - N I L A P A T WS A HELGHAVGMSHDEQ- - - Y Y Q C R G R L - - - - - C

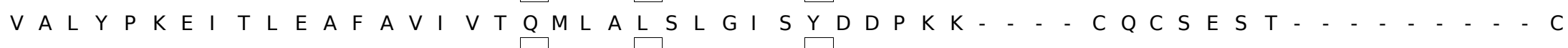
$\checkmark S T D H-S E L P I G A A A T M A H E I G H S L G L S H D P D-\cdots G-C C \vee E A A A E S-\cdots$ VSTDH-SELPI GA A A T A HEI GHSLGLSHDPD- - G - C C VEAAAE - - - G G C VI E A K - K KN N V A L V GVMSHELGHVLGMPDVPF- - N - T K C P S G - C 


\section{ADAMTS1} ADAMTS2ADAMTS2-II ADAMTS3 ADAMTS4 ADAMTS5 ADAMTS6 ADAMTS7 ADAMTS8 ADAMTS9-I ADAMTS9-II ADAMTS9-III ADAMTS10 ADAMTS12 ADAMTS13-I ADAMTS13-II ADAMTS13-III ADAMTS13-IV ADAMTS14-I ADAMTS14-II ADAMTS15 ADAMTS16 ADAMTS17 ADAMTS18-I ADAMTS18-II ADAMTS19 ADAMTS20-I ADAMTS20-II
G L Q A A F T T A HEL G HVF N M P H D A K Q C A S L N G V N Q D - - - S - H

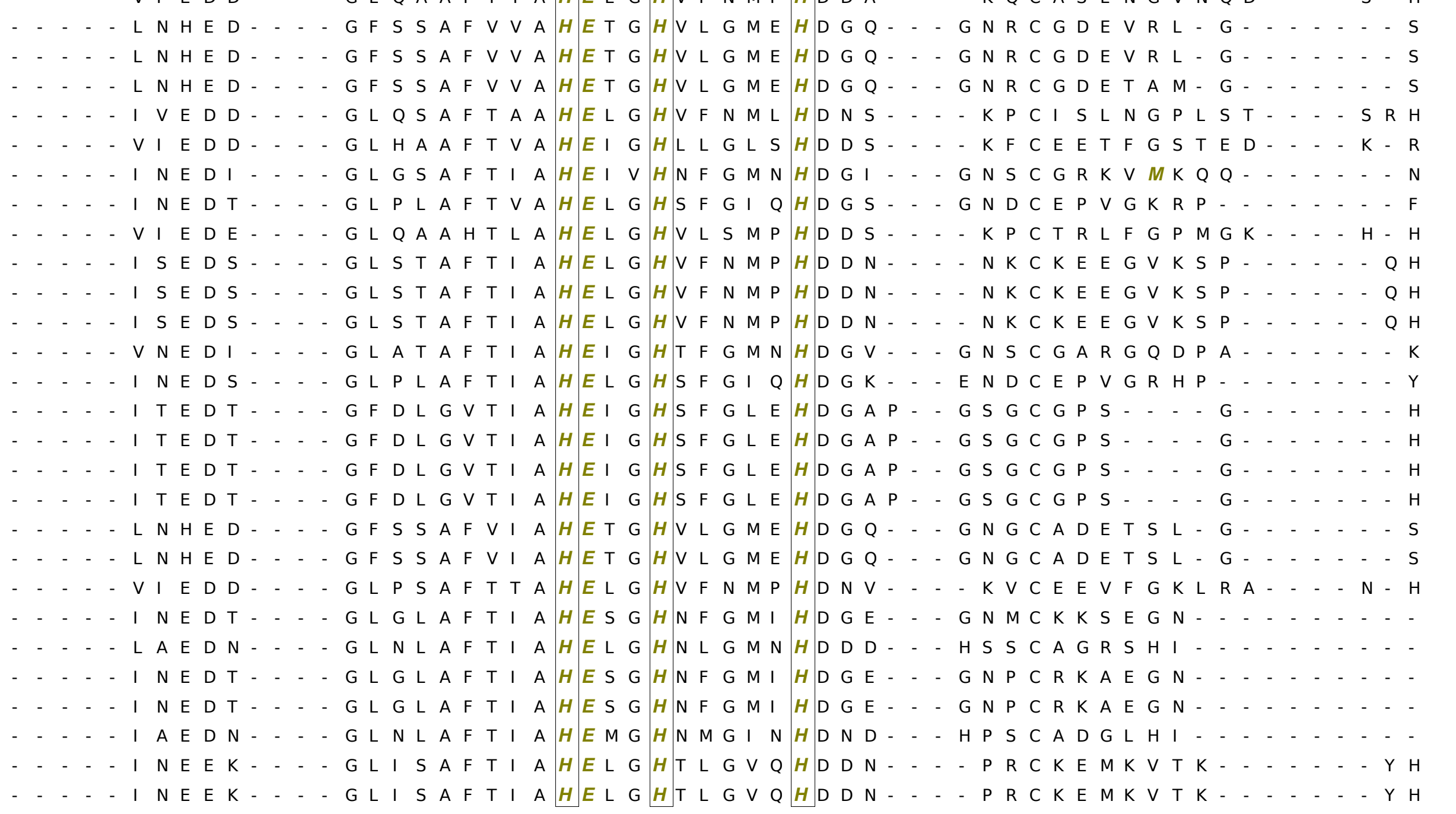


I MNPEA I H- - F S G V K I F S N C S F E D F A H F I S K Q K S Q C L H N Q P $V M D S D G S-\cdots--I P A L K F S K C S Q N Q Y H Q Y L K D Y K P T C M L N I C-$ I MAGS I G - - S S F P R M S D C S Q A Y E S F L E R P S C C L NAP I $M N S G A S-\cdots$ - - - GS R F S C S A E D F K K T L N K G G N C L L I P I MNSGAS - - - G S R N F S C S A E D F E K L T L N K G G N C L L I P I MYARATSGDKLNNNKFSLCSIRN I S Q R L E K K R N N C F V E S G I MEDTG- - - F Y L P R K S R C S I DEYNQFL Q E G G G S C F N K I MEDTG- - - F Y L P R K F R C S I DEYNQFL Q E G G G S C F N K P I $M N A S T G-\cdots Y P F P V F S S C S R K D L E T S L E K G M G V C L F N L P-$ I MNASTG- - Y P F P MVFS SCSRKDLETSLEKGMGVCLFNLPI MEASTD- - F L P G L F S N S R R A L E K A L D GMGSCL FERLI MEASTD- - F L P G L N S NCSRRALEKALLDGMGSCLFERLI MEASTD- - F L P G L F S N C R R A L E K A L L D G M G S C FER L I MEASTD- - F L P G L F S N C R R A L E K A L L G M G S C FER I MEASTD- - F L P G L F S N C R R A L K A L L D G M G C L F E R I MEASTD- - F L P G L F S N C S R A L E K A L L D G M G C L FER L $V M Y P I A V S G D H E N N K M F S N C S K Q S I Y K T I E S K A Q E C F Q E R S-$ $V M Y P I A V S G D H E N N K M F S N C S K Q S I Y K T I E S K A Q E C F Q E R S-$ I MNHEAVS- - A S GRKI F S N S M H D Y R Y F V S K F E T K C L Q K L S I MAAATG- - HPFPKVFNGCNRRELDRYLQSGGGMCLSNMPI MAAATG- - H P F P K F N G C R RELDRYL QSGGGMCLSNMPI MHAYRK- - - - V T T K F S C S Y A Q Y W D S T I S G L C I Q P P P I $M N T F R V-\cdots$ - - P A E K F T N S Y A D F M T T L N Q G - S C H N P I MGDTG- - - Y Y L P K K F T Q C N I E E Y H D F L N S G G G A C L F N K P I MGDTG- - - Y Y Y P K K F T Q C N I E E Y H D F L N S G G G A C L F N $P$ I MGDTG- - - Y Y Y P K K F T Q C N I E E Y H D F L N S G G GACLFNK I MGDTG- - - Y Y Y P K K F T Q C N I E E Y H D F L N S G G G A C F N K P I MGDTG- - - Y Y Y P K K F T Q C N I E E Y H D F L N G G G A C L F N K P I MEETG- - - V S H S R F S K C S L E Y R D F L Q R G GACLFNR P $V M D K A L S--F Y I P T D F S S C S R L S Y D K F F E D K L S N C L F N A P-$ $V M D K A L S-\cdots F Y I P T D F S S C S R L S Y D K F F E D K L S N C L F N A P-$ I $M H E G N P$ - - - - P I TKF S N C S Y G D F W Y T VERT-KCLLETVI $M H E G N P$ - - - - P I T K F S N C S Y G D F W Y T VERT-KCLLETVI MHEGNP- - - - P I TKF S N C S Y G D F WE Y T VERT-KCLLETVI MGS GR - - - - - - T G F N C S Y I S F F K H I S S G - A T C L N I P I $M N P E V V Q--S N G V K T F S S C S L R S F Q N F I S N V G V K C L Q N K P-$ $V M A A A T G-\cdots H P F R V F S A C S R R Q L R A F F R K G G G A C L S N A P-$ $V M A A A T G-\cdots H P P R V F S A C S R R Q L R A F F R K G G G A C L S N A P-$ $V M N Q Y L S--S K F P K D F S T S C R A H F E R Y L L S Q K P K C L L Q A P-$ 
ADAMTS1 ADAMTS2-I ADAMTS2-II ADAMTS3 ADAMTS4 ADAMTS5 ADAMTS6 ADAMTS7 ADAMTS8

ADAMTS9-I

ADAMTS9-II ADAMTS9-III ADAMTS10 ADAMTS12 ADAMTS13-I ADAMTS13-II ADAMTS13-III ADAMTS13-IV ADAMTS14-I ADAMTS14-II ADAMTS15 ADAMTS16 ADAMTS17 ADAMTS18-I ADAMTS18-II ADAMTS19 ADAMTS20-I ADAMTS20-II
M MASMLSNLD- - HS QP WSPCS A Y M I TSFLDNGHGECLMDKPI MAPLVQAAF- - HRFHWSRCSQQELSRYLHS- Y Y C L L D D P I MAPLVQAAF- - HRFHWSRCSQQELSRYLHS- Y PCLLDDP $V M A P L V Q A A F-H R Y H W S R C S G Q E L K R Y I H S-$ - $P D C L L D D P-$ $\checkmark M A P V M A H V D-P E E P W S P C S A R F I T D F L D N G Y G H C L L D K P$ LMS SILTSI D- A SKPWSKCTSATITEFLDDGHGNCLLDLP -

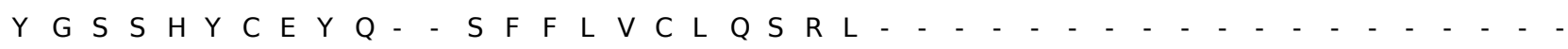
I MS P Q L L Y D A - A P L TWSRCSR QY I TRFLDRGWGLCLDDPP VMAPLFVHLN- - QTLPWSPCSAMYLTELLDGGHGDCLLDAPVMAPTLNFYT- N N WMWSKCSRKYITEFLDTGYGECLLNEP $V M A P T L N F Y T-N P W M W S K C S R K Y I T E F L D T G Y G E C L L N E P$ VMAPTLNFYT- - NPWMWSKCSRKYITEFLDTGYGECLLNEPLMAAHITMKT- N P F V WS SCSRDYITSFLDSGLGLCLNNRPI MSR QLQYDP- - TPLTWSKCSEEYITRFLDRGWGFCLDDI P $V M A S D G A A P R$ - A G L A WS PCSRR QLLSLLSAGRAR C VWDPP. $V M A S D G A A P R$ - - A GLAWSPCSRRQLLSLLSAGRARCVWDPPVMASDGA APR - A GLAWSPCSRRQLLSLLSANEQC - . - . $\checkmark M A S D G A A P R \ldots A G L A W S P C S R R Q L L S L L R P \ldots$ $V M A P L V Q A A F-H R F H W S R C S K L E L S R Y L P S-$ - Y D CLLDDP $V M A P L V Q A A F-H R F H W S R C S K L E L S R Y L P S-$ - $D C L L D D P-$ MMSPTLI QI D- - R A N P WS ACSAAII T DFLDSGHGDCLLDQPI MSPTLAGRN- - GVFSWSPCSR QYLHKFLSTAQA I CLADQP. MS GEWVKGRNP-SDLSWS SCSRDDLENFLKSKVSTCLLVTDP I MSPTLTGNN- - GVFSWS SCSR QY LKKFLSTPQAGCLVDEPI MSPTLTGNN- - GVFSWS SCSR QYLKKFLSTPQAGCLVDEP$M S G E W I K G Q N L-G D V S W S R C S K E D L E R F L R S K A S N C L L Q T N-$ VMAPALSFHM- - S P WS WS NCSRKYVTEFLETGYGECLLDKP VMAPALSFHM- - SPWSWSNCSRKYVTEFLETGYGECLLDKP- 
Figure 2S

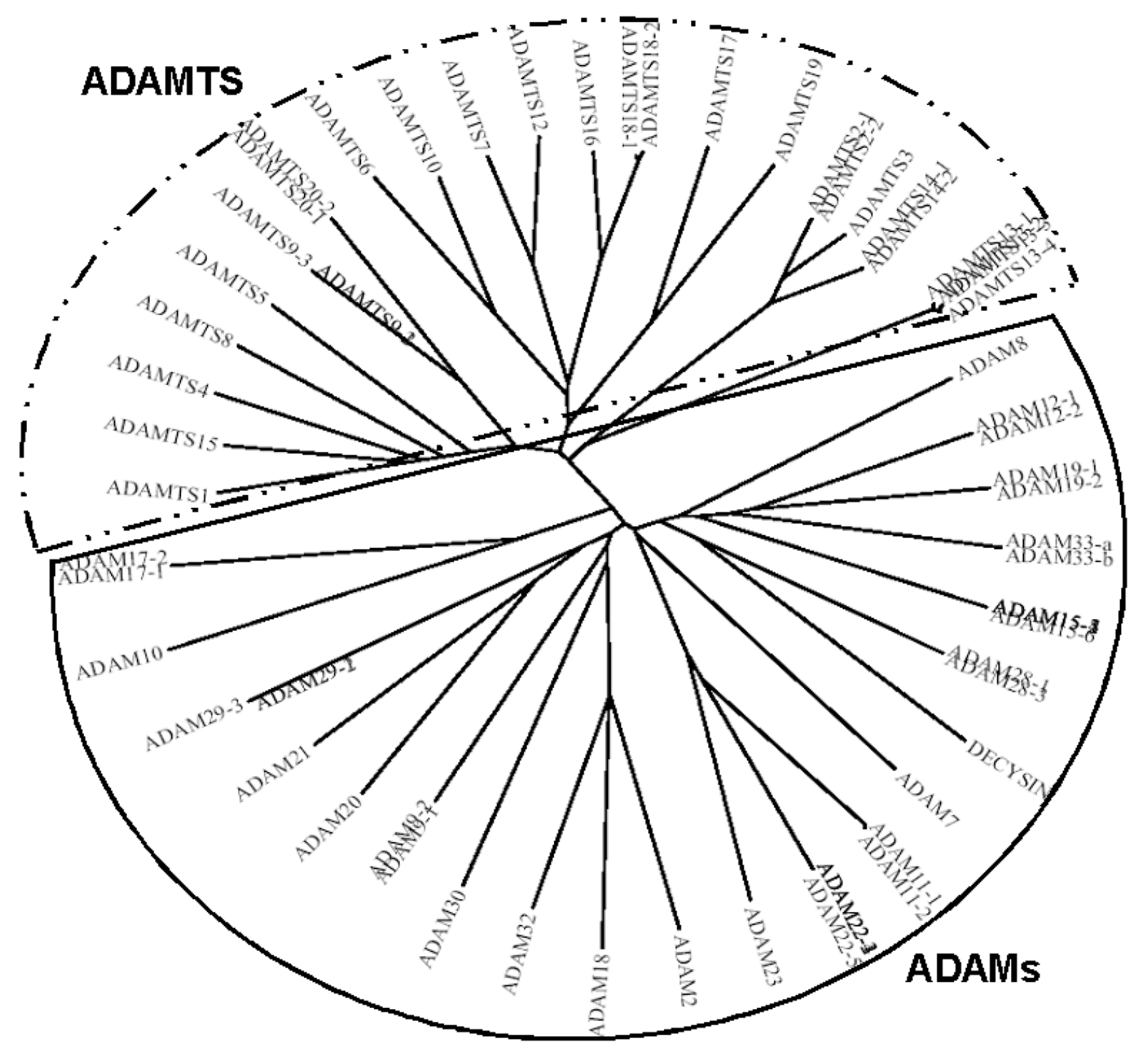


Figure 3S

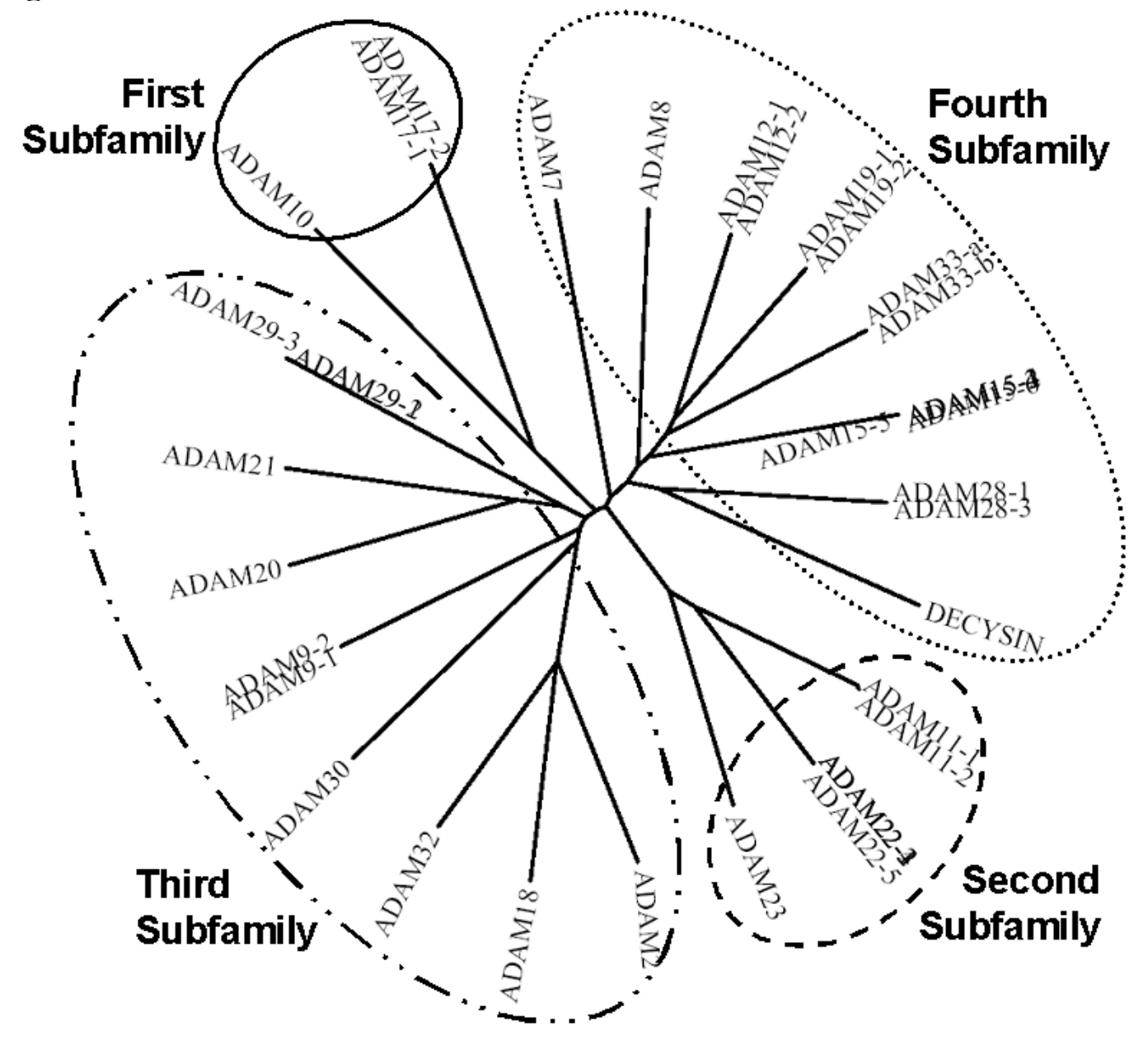


Figure 4S

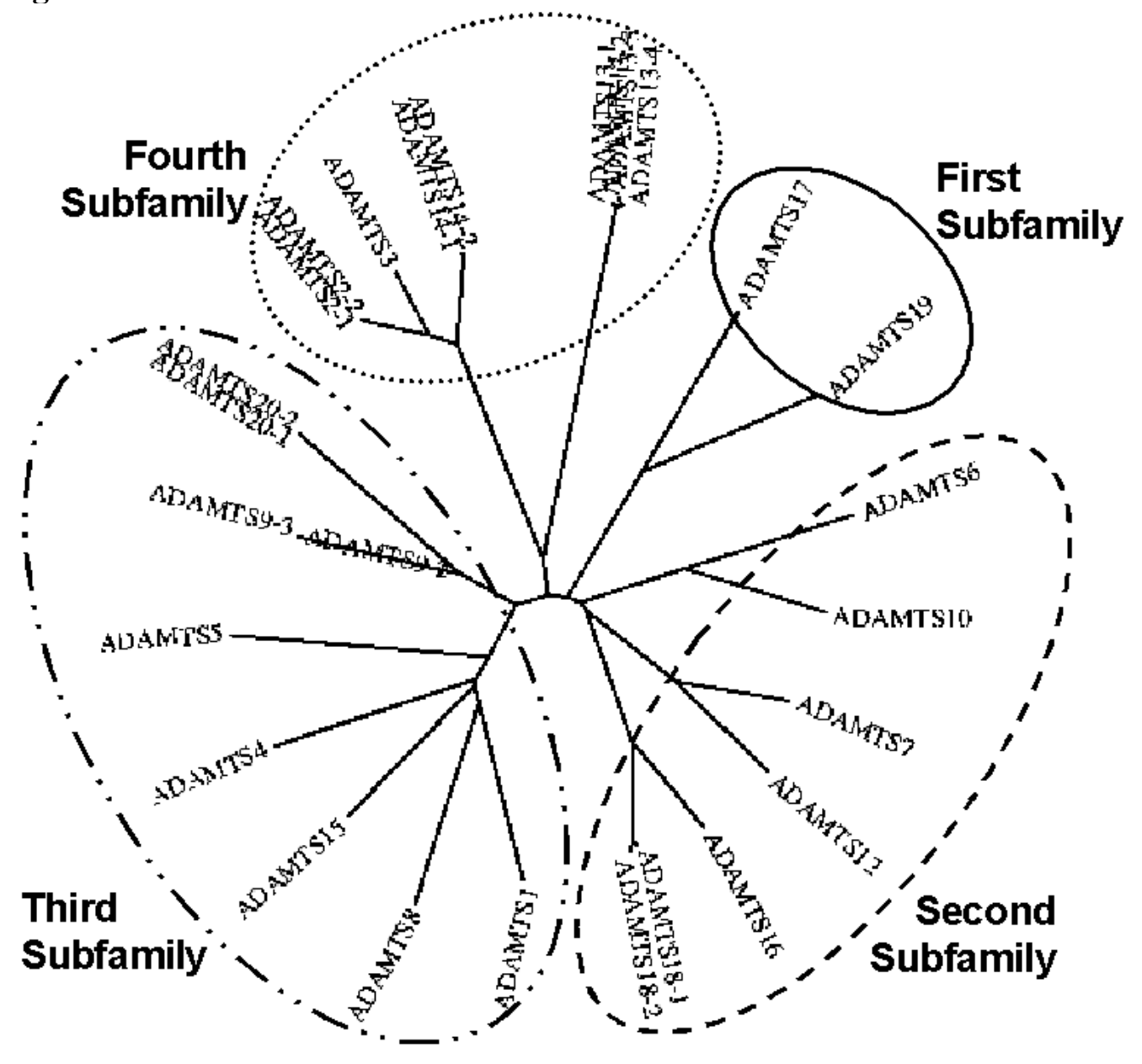

